\title{
Microtomografia de Raios X (microCT) Aplicada na Caracterização Anatômica da Madeira de Folhosa e de Conífera
}

\author{
Ugo Leandro Belini ${ }^{1}$, Mario Tomazello Filho ${ }^{1}$, Vinícius Resende de Castro ${ }^{1}$, \\ Graciela Ines Bolzon de Muniz², Paulo Renato Orlandi Lasso ${ }^{3}$, \\ Carlos Manoel Pedro Vaz ${ }^{3}$ \\ ${ }^{1}$ Departamento de Ciências Florestais, Escola Superior de Agricultura "Luiz de Queiroz" - ESALQ, \\ Universidade de São Paulo - USP \\ ${ }^{2}$ Universidade Federal do Paraná - UFPR \\ ${ }^{3}$ Embrapa-CNPDIA
}

\begin{abstract}
RESUMO
A microtomografia computadorizada de raios X (microCT) é uma das mais avançadas técnicas de análise não destrutiva e possibilita a reconstrução da estrutura anatômica da madeira e de seus produtos. O trabalho aplicou a microCT na análise da anatomia da madeira de Amburana cearensis e de Pinus sp. A microCT possibilitou a obtenção de imagens da estrutura macro e microscópica da madeira das duas espécies, permitindo a sua caracterização, descrição e comparação com a da literatura. As vantagens da microCT, em relação à confecção de lâminas histológicas, mantendo a integridade das amostras de madeira, painéis, carvão, papéis, etc. são discutidas.
\end{abstract}

Palavras-chave: microtomografia de raios X, Amburana cearensis, Pinus sp.

\section{X-ray Microtomography to Anatomical Characterization of Softwood and Hardwood}

\begin{abstract}
$\mathrm{X}$-ray computed microtomography (microCT) is one of the most advanced non-destructive techniques enabling the reconstruction of wood anatomical structure and its products. This work applied the microCT to the analysis of wood anatomy of Amburana cearensis and Pinus sp. Through microCT it was possible to obtain images of the macro and microscopic wood structure of the two species, followed by the characterization, description and comparison to the literature. The advantages of microCT are discussed in relation to the preparation of histological slides, providing the integrity of wood, panels, charcoal, paper samples, etc.
\end{abstract}

Keywords: X-ray microtomography, Amburana cearensis, Pinus sp.

\section{INTRODUÇÃO}

A microtomografia computadorizada de raios $\mathrm{X}$ (microCT) fundamenta-se na atenuação de feixes de raios $\mathrm{X}$ que, ao atravessar um objeto em análise em diversos ângulos, possibilita a reconstrução da sua estrutura interna. Como técnica não destrutiva, apresenta inúmeras vantagens na análise da madeira 
e de seus produtos, mantendo a integridade das amostras e dispensando o laborioso processo laboratorial de confecção de lâminas histológicas (Steppe et al., 2004; Wernersson et al., 2009).

A microCT constitui-se em uma das mais avançadas tecnologias de análise não destrutiva, proporcionando a construção de imagens da estrutura interna em 2D e 3D (Mayo et al., 2010). A microCT está sendo aplicada, notadamente na presente década, na análise anatômica da madeira (Steppe et al., 2004; Van den Bulcke et al., 2009a; Trtik et al., 2007) e de seus produtos, como painéis (Lux et al., 2006) e carvão vegetal (Bird et al., 2008).

No entanto, a microCT apresenta-se como importante e potencial metodologia para a caracterização da qualidade da madeira, dos anéis de crescimento do lenho, dos defeitos do lenho resultantes do crescimento, do ataque de organismos xilófagos à madeira, etc. (Wu \& Peng, 2007; Van den Bulcke et al., 2009b; Trtik et al., 2007).

Neste contexto, o presente trabalho teve como objetivo aplicar no Brasil a metodologia de microCT como nova linha de pesquisas em madeira e produtos dela, através da análise da estrutura anatômica microscópica do lenho de folhosa (Amburana cearensis, cerejeira) e de conífera (Pinus sp., pinus).

\section{MATERIAL E MÉTODOS}

\subsection{Espécies florestais}

Para o presente estudo, foram selecionadas amostras de madeira de Amburana cearensis (Fr. All.) A. C. Smith, cerejeira e de Pinus sp., pinus ou pinheiro, coletadas na Xiloteca do Departamento de Ciências Florestais da ESALQ/USP, Piracicaba, SP.

\subsection{Preparo das amostras de madeira}

Das amostras de madeira das duas espécies, foram cortados corpos de prova cilíndricos com as dimensões de $20 \times 20 \mathrm{~mm}$ (diâmetro da seção transversal $x$ altura) (Figura 1a).

\subsection{Aplicação da microCT na análise da madeira}

\subsubsection{O microtomógrafo de raios $X$}

Para a obtenção das imagens tomográficas da madeira foi utilizado um Microtomógrafo de Raios X, marca SkyScan, modelo 1172, do Laboratório de Técnicas Nucleares da Embrapa Instrumentação Agropecuária/CNPDIA, em São Carlos, SP. O equipamento possui como características técnicas,

i) tubo de raios $\mathrm{X}$ : tensão de $100 \mathrm{kV}$ e corrente de $100 \mu \mathrm{A}$;

ii) câmera digital R-X 12-bit;

iii) três posições automáticas de filtro para seleção de energia;

iv) tamanho pixel detector $<0,8 \mu \mathrm{m}$;

v) detalhes de detecção $<1 \mu \mathrm{m}$;

vi) resolução espacial de $5 \mu \mathrm{m}$;

vii) comprimento máximo do objeto $=100 \mathrm{~cm}$; e diâmetro máximo $=7 \mathrm{~cm}$ (SkyScan, 2010).

\subsubsection{Princípio de funcionamento}

Os corpos de prova, cilíndricos de madeira, foram acondicionados $\left(20{ }^{\circ} \mathrm{C} ; \quad 50 \% \quad \mathrm{UR} ; 12\right.$ horas), transferidos para o interior da câmara de irradiação e fixados no sistema de movimentação do equipamento. No processamento da análise microtomográfica, os raios $\mathrm{X}$ emitidos pela fonte (tubo de raios $\mathrm{X}$ ) atravessam a amostra de madeira, no sentido longitudinal, a distâncias de $9 \mu \mathrm{m}$, com a radiação não atenuada captada por um intensificador de imagens e, em seguida, por um detector (câmera CCD) (Figura 2).

\subsubsection{Obtenção das imagens tomográficas}

As imagens tomográficas das seções transversais dos corpos de prova de madeira foram obtidas em 2 etapas, sendo (i) aquisição das imagens radiográficas de diversas projeções angulares da amostra de madeira em rotação de 180 ou $360^{\circ}$, com passos de rotação precisos e (ii) reconstrução das imagens das seções transversais da amostra de madeira a partir das imagens das projeções angulares, por meio do algoritmo de reconstrução de feixe cônico de Feldkamp modificado, instalado no cluster de computadores (Lasso et al., 2008). 


\subsubsection{Análise da estrutura anatômica da madeira}

A imagem da estrutura anatômica das seções da madeira é projetada na tela do monitor utilizando o software DataViewer. Pela movimentação do
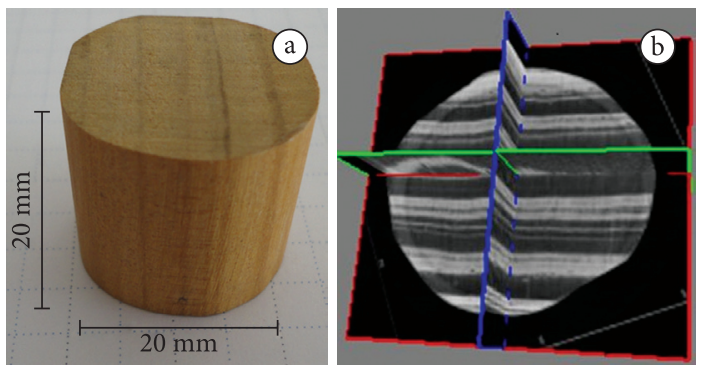

Figura 1. a) Corpo de prova de madeira de Pinus sp. utilizado na obtenção de imagens da estrutura anatômica por microCT; b) Imagem da seção transversal da estrutura anatômica da madeira, por microCT e indicação das linhas de referência para a projeção dos planos longitudinais.

Figure 1. a) Sample of Pinus sp. wood used to obtain images of anatomical structure by microCT; b) Image of cross section of the wood anatomical structure, for microCT, indicating the reference lines for projection the longitudinal planes. cursor, as duas linhas de orientação (verde e azul) (Figura 1b) percorrem o eixo longitudinal (ao longo da altura) da amostra de madeira analisada, visualizando-se as respectivas imagens sequenciais microscópicas dos planos transversal e longitudinais radial e tangencial, permitindo a reconstrução da estrutura anatômica da madeira em 2D, através da sobreposição das imagens. Posteriormente, com o software DataViewer, faz-se nova sobreposição para reconstrução das imagens da estrutura anatômica da madeira em formato $3 \mathrm{D}$.

\section{RESULTADOS E DISCUSSÃO}

As imagens da estrutura anatômica do plano transversal e longitudinais radial e tangencial da madeira de Amburana cearensis e de Pinus sp., obtidas através da análise por microCT, possibilitam a sua caracterização macro e microscópica (Figuras 3, 4). A movimentação das linhas de referência perpendiculares entre si, ao longo da altura da amostra de madeira, permite a reconstrução da disposição e do alinhamento dos seus elementos anatômicos em $2 \mathrm{D}$ e 3D.

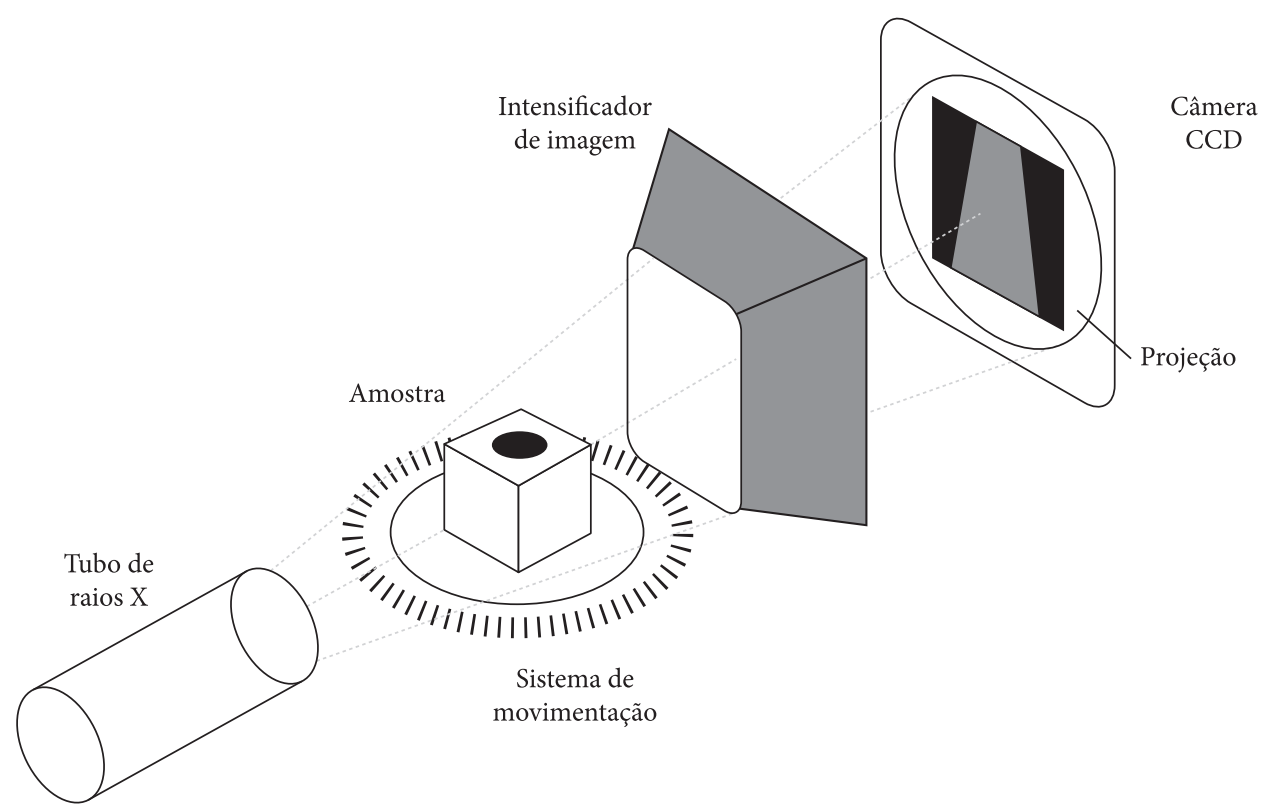

Figura 2. Princípio de funcionamento e componentes básicos do microCT, indicando o tubo de raios $\mathrm{X}$, posicionamento da amostra de madeira no sistema de movimentação na câmara de irradiação, intensificador de imagem e projeção da imagem da estrutura anatômica coletada em câmera CCD (adaptado de Pereira, 2009).

Figure 2. Operating principle and basic components of microCT, indicating the $\mathrm{x}$-ray tube, positioning the sample timber handling system in the irradiation chamber, image intensifier and image projection of the anatomical structure collected in CCD camera (adapted from Pereira, 2009). 
A análise da estrutura anatômica macro e microscópica da madeira de Amburana cearensis nos planos transversal e longitudinais radial e tangencial possibilita a visualização dos vasos, do parênquima longitudinal e radial e das fibras (Figura 3a,b,c). A estrutura anatômica microscópica permite analisar e caracterizar os vasos (solitários predominantes e múltiplos, circulares e ovais, alguns obstruídos por tiloses), parênquima longitudinal (contrastado, abundante, aliforme e vasicêntrico, confluente interligando 2-3 vasos, com a presença de cristais indicados por pontos brancos), parênquima radial (finos, numerosos, irregularmente espaçados) e fibras (Figura 4a). A caracterização anatômica da madeira de Amburana cearensis por microCT é corroborada com a descrita na literatura (Mainieri \& Pereira, 1965; Mainieri \& Chimelo, 1989).

A diferenciação e o contraste verificados no lenho de Amburana cearensis devem-se às dimensões transversais (largura, diâmetro do lume e espessura da parede) das células dos tecidos do lenho e à intensidade de atenuação dos feixes de raios $\mathrm{X}$ que percorrem longitudinalmente a amostra do lenho a cada $9 \mu \mathrm{m}$. A elevada precisão das leituras possibilita que as células do parênquima longitudinal e radial e o lume dos vasos (principalmente) apresentemse de coloração mais escura pela menor atenuação dos feixes de raios X. Os distintos e característicos pontos esbranquiçados nas células do parênquima longitudinal indicam a presença de cristais, de maior densidade; da mesma forma, a parede dos vasos e as substâncias químicas constituintes das tiloses que preenchem o seu lume são de coloração mais clara, com limites definidos pela elevada atenuação dos raios $\mathrm{X}$ no processo de leitura do microCT. Ainda, na Figura 3 para o lenho de Amburana cearensis, a coloração clara do tecido de sustentação (fibras) é indicativa de fibras estreitas, parede espessa e de lume reduzido. A literatura relata que a obstrução dos vasos por tilose, gomas, etc. diminuem a tratabilidade, a permeabilidade e a movimentação capilar da água na madeira e processos de secagem (Klitzke et al., 2008).

A análise da estrutura anatômica macro e microscópica da madeira de Pinus sp. nos planos transversal e longitudinais radial e tangencial possibilita a visualização dos anéis de crescimento, formados pelos lenhos inicial e tardio, além de falsos anéis de crescimento, finas linhas do parênquima radial e pequenos pontos indicativos dos canais de resina (Figura 3a,b,c'). A estrutura anatômica microscópica da madeira de Pinus sp. permite analisar e caracterizar um anel de crescimento anual completo com os respectivos lenhos inicial, de transição e lenho tardio e finas linhas de anéis de crescimento falsos, além de dois anéis de crescimento anuais incompletos. Os canais de resina são distintos e localizados em finas linhas de traqueídes de lenho tardio falso, com poucos no lenho inicial; os raios são representados pelas finas e frequentes linhas paralelas perpendiculares aos anéis de crescimento (Figura 4b). A caracterização anatômica da madeira de Pinus sp. por microCT é corroborada com a descrita na literatura (Panshin \& De Zeeuw, 1970; Ferreira, 2009)

Conforme verificado para a madeira de folhosa, a diferenciação e o contraste dos lenhos inicial e tardio nos anéis de crescimento do lenho de Pinus sp. devem-se à diferença da intensidade de atenuação dos feixes de raios $\mathrm{X}$. As células de traqueídes do lenho inicial e dos anéis de crescimento de lenho inicial falsos (com traqueídes de maior largura radial, parede fina, maior lume), bem como o lume dos canais de resina, apresentam-se de coloração mais escura pela menor atenuação dos feixes de raios X. Por outro lado, as traqueídes de lenho tardio e dos anéis de crescimento de lenho tardio falsos (com traqueídes de menor largura radial, parede espessa, menor lume), bem como as células epiteliais dos canais de resina, de maior densidade, são de coloração mais clara, com limites definidos pela elevada atenuação dos raios $\mathrm{X}$. Os valores de densidade dos lenhos inicial e tardio dos anéis de crescimento de Pinus caribaea var. hondurensis, obtidos por densitometria de raios $\mathrm{X}$, são da ordem de 02-05 g.cm ${ }^{-3}$ e 08-1,2 g.cm ${ }^{-3}$, respectivamente, corroborando as observações realizadas através da microCT (Ferreira \& Tomazello Filho, 2009).

A qualidade das imagens da estrutura anatômica da madeira de Amburana cearensis e de Pinus sp., obtidas através da microCT, permite a avaliação quantitativa das dimensões e porcentagens dos tecidos e células constituintes do lenho, com a aplicação de softwares específicos. Como exemplo: a determinação da área de vasos em madeiras de folhosas e sua relação com os fenômenos de 
transporte de fluídos na madeira durante o processo de secagem (Wu \& Peng, 2007). Da mesma forma, a microCT é uma ferramenta importante na análise da dinâmica do ataque de fungos xilófagos na madeira associado com a avaliação das suas características anatômicas (Van den Bulcke et al., 2009b).
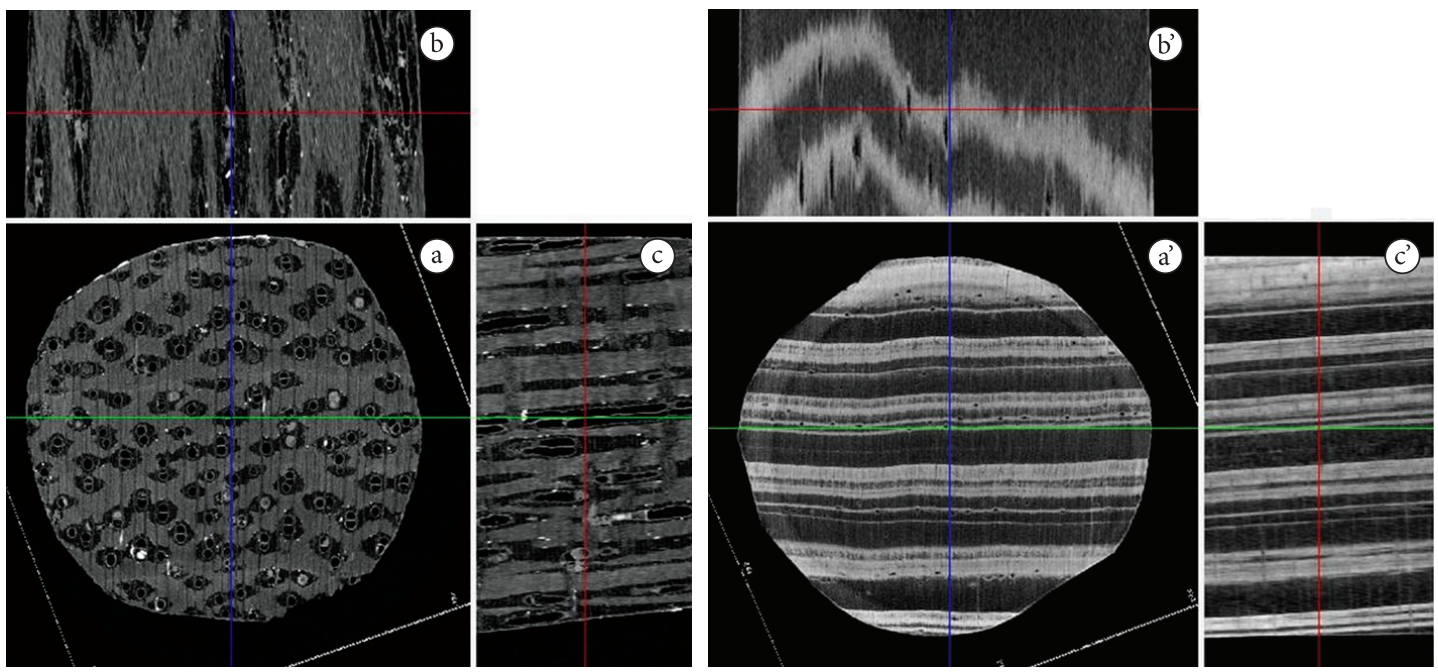

Figura 3. Estrutura anatômica do plano transversal (a, a') e dos respectivos planos longitudinais tangencial (b, b') e radial (c, c') da madeira de Amburana cearensis e de Pinus sp., respectivamente. As duas linhas de orientação cruzada percorrem o eixo longitudinal da amostra de madeira projetando as imagens sequenciais dos planos transversal e longitudinais radial e tangencial.

Figure 3. Anatomical structure of the transverse plane (a, a') and its tangential longitudinal planes (b, b') and radial (c, c') from Amburana cearensis and Pinus sp. wood, respectively. The two guidelines, cross traverse the longitudinal axis of the wood sample by projecting the sequential images of transverse and longitudinal planes radial and tangential.
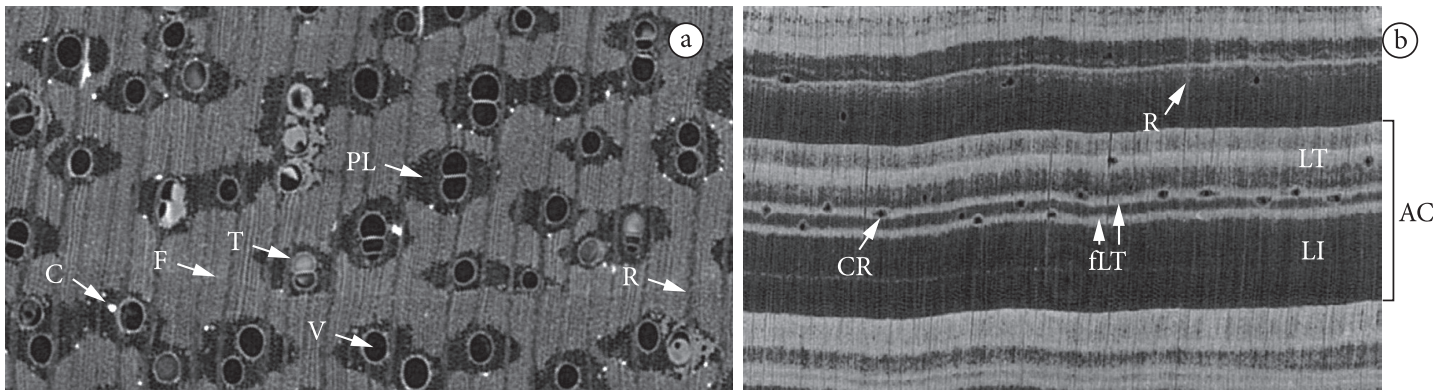

Figura 4. Estrutura anatômica microscópica do plano transversal obtida através de imagens em microCT. a) Amburana cearensis, indicando o parênquima longitudinal (PL) com cristais (C), parênquima radial (R), vasos (V) obstruídos por tilose (T) e fibras (F). b) Pinus sp., indicando o anel de crescimento (AC) com os lenhos inicial (LI) e tardio (LT), faixas de lenho tardio (fLT) com canais de resina (CR) e parênquima radial (R).

Figure 4. Microscopic anatomical structure of the transverse plane images obtained by microCT. a) Amburana cearensis, indicating the longitudinal parenchyma (PL) with crystals (C), parenchyma (R), vessels (V) blocked by tylosis (T) and fiber (F). b) Pinus sp., indicating the growth ring (AC) earlywood (LI) and latewood (LT), bands of latewood (FLT) with the resin canals (RC) and parenchyma (R). 
A aplicação da microCT apresenta inúmeras vantagens, como método não destrutivo, em relação ao processo de confecção de lâminas histológicas. A microCT permite, ainda, a avaliação quantitativa da estrutura anatômica da madeira, a reconstrução da disposição dos elementos anatômicos em 3D e a análise não destrutiva de painéis, carvão vegetal e demais produtos de madeira.

\section{STATUS DA SUBMISSÃO}

Recebido: 03/12/2010

Aceito: 13/03/2011

Resumo publicado online: 15/03/2011

Artigo completo publicado: 31/03/2011

\section{AUTOR (ES) PARA CORRESPONDÊNCIA}

\section{Ugo Leandro Belini}

Departamento de Ciências Florestais, Escola Superior de Agricultura

"Luiz de Queiroz - ESALQ", Universidade de São Paulo - USP, Av. Pádua Dias, 11, CP 09, Piracicaba, SP, Brasil e-mail: ulbelini@esalq.usp.br

\section{AGRADECIMENTOS}

Os autores expressam seus agradecimentos à FAPESP, à EMBRAPA-CNPDIA e ao Laboratório de Anatomia, Identificação e Densitometria de Raios X em Madeira do Departamento de Ciências Florestais da ESALQ/USP.

\section{REFERENNCIAS}

Bird MI, Ascough PL, Young IM, Wood CV, Scott AC. $\mathrm{X}$-ray microtomographic imaging of charcoal. Journal of Archaeological Science 2008; 35:2698-2706. http:// dx.doi.org/10.1016/j.jas.2008.04.018

Ferreira ATB. Caracterização da estrutura anatômica do lenho, dos anéis de crescimento e dos canais de resina de árvores de Pinus caribaea var. hondurensis Barr. et Golf. [dissertação]. Piracicaba: Escola Superior de Agricultura "Luiz de Queiroz", Universidade de São Paulo; 2009.

Ferreira ATB, Tomazello Filho M. Caracterização dos anéis de Crescimento de árvores de Pinus caribaea var. hondurensis Barr. et Golf. por densitometria de raios X. Scientia Forestalis 2009; 37(83):287-298.

Klitzke RJ, Savioli DL, Muñiz GIB, Batista DC. Caracterização dos lenhos de cerne, alburno e transição de jatobá (Hymenaea sp.) visando ao agrupamento para fins de secagem convencional. Scientia Forestalis 2008; 36(80):279-284.

Lasso PRO, Vaz CMP, Naime JM. Recomendações para utilização do microtomógrafo de raios-X Sky Scan 1172 em ciências agrárias e biológicas. Documentos 372008.

Lux J, Delisée C, Thibault X. 3D characterization of wood based fibrous materials: an application. Image Analysis \& Stereology 2006; 25:25-35.

Mainieri C, Pereira JA. Madeiras do Brasil: sua caracterização macroscópica, usos comuns e índices qualitativos, físicos e mecânicos. São Paulo: Instituto de Pesquisas Tecnológicas; 1965.

Mainieri C, Chimelo JP. Fichas de características das madeiras brasileiras. São Paulo: Instituto de Pesquisas Tecnológicas; 1989.

Mayo S, Chen F, Evans R. Micron-scale 3D imaging of wood and plant microstructure using high-resolution $\mathrm{X}$-ray phase-contrast microtomography. Journal of Structural Biology 2010; 171(2):182-188. http://dx.doi. org/10.1016/j.jsb.2010.04.001

Panshin AJ, De Zeeuw C. Text book of wood technology. $3^{\text {rd }}$ ed. New York: McGraw-Hill, 1970.

Pereira SF. Porosidade do solo avaliada pela análise de imagens com os softwares NOESIS Visilog, CTAn, Image J, Scion Image e Imago [dissertação]. Botucatu: Faculdade de Ciências Agronômicas, Universidade Estadual Paulista; 2009.

Skyscan. SkyScan1172 high-resolution micro-CT. [cited 2010 out. 21]. Available from: http://www.skyscan.be/ products/1172.htm.

Steppe K, Cnudde V, Girard C, Lemeur R, Cnudde JP, Jacobs P. Use of X-ray computed microtomography for non-invasive determination of wood anatomical characteristics. Journal of Structural Biology 2004; 148:11-21. http://dx.doi.org/10.1016/j.jsb.2004.05.001

Trtik P, Dual J, Keunecke D, Mannes D, Niemz P. 3D imaging of microstructure of spruce wood. Journal of Structural Biology 2007; 159(1):46-55. http://dx.doi. org/10.1016/j.jsb.2007.02.003

Van den Bulcke J, Boone M, Van Acker J, Stevens M, Van Hoorebeke L. X-ray tomography as a tool for detailed anatomical analysis. Annals of Forest Science 2009a; 66(5):508. http://dx.doi.org/10.1051/forest/2009033

Van den Bulcke J, Boone M, Van Acker J, Van Hoorebeke, L. Three-dimensional X-ray imaging and analysis of fungi on and in wood. Microscopy and Microanalysis 2009b; 15(5):395-402. http://dx.doi.org/10.1017/ S1431927609990419 
Wernersson ELG, Brun A, Hendriks CLL. Segmentation of wood fibres in 3D CT images using graph cuts. Lecture Notes in Computer Science 2009; 5716:92-102. http://dx.doi.org/10.1007/978-3-642-04146-4_12
Wu D, Peng XF. Investigation of water migration in porous material using micro-CT during wetting. Heat Transfer - Asian Research 2007; 36(4):198-207. http:// dx.doi.org/10.1002/htj.20156 